\title{
Illustrated Key to the Genera of Free-Living Marine Nematodes in the Superfamily Chromadoroides Exclusive of the Chromadoridae ${ }^{1}$
}

\section{A. C. Tarjan and E. J. Keppner ${ }^{2}$ \\ Identification}

This is an illustrated key to 55 genera of free-living marine nematodes of the superfamily Chromadoroidea, exclusive of the Chromadoridae, (Nematoda: Chromadorida: Chromadorina).

Illustrations are almost all limited to the heads and caudal regions of the included genera. Specific anatomical features are designated to facilitate use of the key.

This is the fourth in a series of keys by the authors to the groups of the free-living marine nematodes. The purpose of these keys is to provide students and other interested persons with a tool to begin the identification of free-living marine nematodes. These keys, along with those provided by Tarjan (1980) and Platt and Warwick (1983), should provide a basis for the identification of the genera of free-living marine nematodes.

Hope and Murphy (1972), Gerlach and Riemann (1974), Andrassy (1976), Lorenzen (1981), and Inglis (1983) have published classifications of free-living marine nematodes. Platt and Warwick
(1988) provided descriptions and figures for the species of chromadorids reported from Britain.

The key presented herein is not intended to reflect phylogenetic relationships. It includes 55 genera of free-living marine nematodes in the suborder Chromadorina, superfamily Chromadoroidea, exclusive of the family Chromadoridae.

Although the key follows the classification of Lorenzen (1981), Jensen (1979) and Platt (1982) should be consulted regarding the Comesomatidae and Ethmolaimidae. Jensen (1979) revised the Comesomatidae and provided keys to the subfamilies, genera, and species. Lorenzen (1981) recognized the family Neotonchidae. Platt (1982) revised the family Ethmolaimidae and included the neotonchids in the Ethmolaimidae as the subfamily Neotonchinae Wieser \& Hopper, 1966. Platt (1982) provided keys to the subfamilies, genera, and species of the Ethmolaimidae. Platt and Warwick (1988) also included the neotonchids in the Ethmolaimidae but did not segregate taxa at the subfamily level.

1. This document is EENY-082, one of a series of Featured Creatures from the Entomology and Nematology Department, Florida Cooperative Extension Service, Institute of Food and Agricultural Sciences, University of Florida. Published: August 1999. This document is also available on Featured Creatures Website at http://creatures.ifas.ufl.edu. Please visit the EDIS Website at http://edis.ifas.ufl.edu. Additional information on these organisms, including many color photographs, is available at the Entomology and Nematology Department WWW site at http://entnemdept.ifas.ufl.edu/.

2. A. C. Tarjan and E. J. Keppner, Entomology and Nematology Department, University of Florida, Gainesville, Florida 32611.

The Institute of Food and Agricultural Sciences is an equal opportunity/affirmative action employer authorized to provide research, educational information and other services only to individuals and institutions that function without regard to race, color, sex, age, handicap, or national origin. For information on obtaining other extension publications, contact your county Cooperative Extension Service office. Florida Cooperative Extension Service/Institute of Food and Agricultural Sciences/University of Florida/Christine Taylor Waddill, Dean. 


\section{Use of the Key}

Couplet numbers and lower case letters refer to the location of related lower-ranked taxa. When one arrives at a family or subfamily name, rather than another couplet, the genera within that taxon immediately follow in couplets designated by lower-case letters. The parenthetical part of each couplet designation indicates the referent couplet, allowing one to work backward, as well as forward, through the key.

Illustrations in the key were obtained from published references. In some cases, portions of the anatomy shown on the drawings are labeled or emphasized with ink; in other cases pertinent anatomical details have been deleted to better portray the structures of interest. Credit is given for each illustration used. (Table 1).

Taxa Included in the Key (Figure 1).

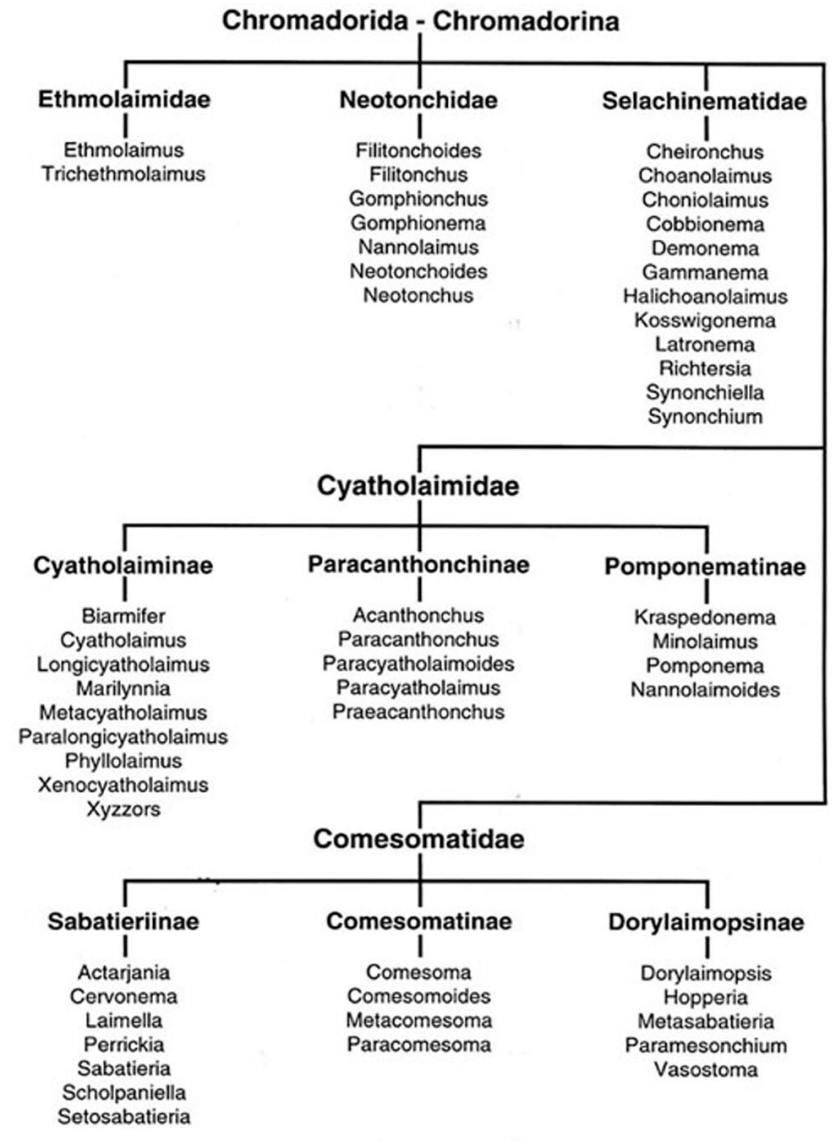

Figure 1. Key.

\section{Abbreviations Used in the Key}

a amphid

be buccal cavity

cs cephalic sensilla

eb esophageal bulb

g gubernaculum

ga gubernacular apophysis

Ip longitudinal punctations

ls labial sensilla

m mandible

ps precloacal supplement

$\mathbf{r}$ rugae

rs cervical sensilla

s spicule

sp small spines

ss somatic sensilla

t teeth

\section{Key to the Genera (exclusive of the Chromadoridae)}

Chromadoroidea. Cuticle with punctations; transverse striations present or absent. Head without helmet. Amphid variable in shape, difficult to discern in some species. Only those species with a spiral or circular amphid included in the key.

[Note: When two consecutive numbers are shown, they refer to the anterior and posterior ends of the nematode.]

1. Amphid a distinct spiral (Figs. 53a, 81a) ... 2

1'. Amphid not a distinct spiral ....... Chromadoridae (not included)

2 (1). Anterior part of buccal cavity with 12 rugae (Fig. 13r); females with reflexed ovaries . . . . 5 
$2^{\prime}$. Anterior part of buccal cavity without 12 rugae; females with outstretched ovaries . . . . . . . . Comesomatidae ............ 3

3 (2). Posterior part of buccal cavity weakly cuticularized; spicules short ( 2 anal body diameters or less) (Fig. 6s); gubernaculum with dorso-caudally directed apophyses (Fig. 4ga) ..... . Sabatieriinae

a. Sublateral longitudinally orientated rows of long cervical sensillae present (Figs. 102, 103) . . . . . ..........Setosabatieria Platt, 1985

a'. Sublateral longitudinally orientated rows of long cervical sensillae absent. . . . . . . . b b

b(a). Amphids situated about 2.0 head diameters posterior to the anterior end (Fig. 7a) outer labial and cephalic sensillae equal in length (Figs. 7cs, 8) . . . . . ......... Cervonema Wieser, 1954

b'. Amphids situated about 1.0 head diameter posterior to anterior end; outer labial and cephalic sensillae equal or unequal in length . . . . . . c

c(b). Outer labial and cephalic sensillae equal in length; amphid with more than 4 spirals (Figs. 89a, 90) . . . . . . . . Pierrickia Vitiello, 1970

$c^{\prime}$. Outer labial and cephalic sensillae unequal in length; amphid with less than 4 spirals . . . . . . d

d(c). Outer labial sensillae shorter than cephalic sensillae; cuticle with lateral differentiation (Figs. 3cs, 4) . . . . . . . . . Actarjania Hopper, 1967

d'. Outer labial sensillae longer than cephalic sensillae; cuticle without lateral differentiation . . . . e

e(d). Outer labial and cephalic sensillae close together; cuticle without distinct punctations (Figs. $49,50)$. . . . . . . . . . . . Laimella Cobb, 1920

e'. Outer labial and cephalic sensillae well separated; cuticle with distinct punctations . . . . f

f(e). Gubernacular apophyses long, directed caudally or dorso-caudally (Figs. 98, 99ga) . . . . . Sabatieria De Rouville, 1903

f'. Gubernacular apophyses short, directed dorsally (Figs. 100, 101ga) . . . . . . . . . . . . . . Scholpaniella Sergeeva, 1979
3'. Posterior part of buccal cavity heavily or moderately cuticularized; spicules elongate (more than 2.0 anal diameters long) (Fig. 18s); gubernacular apophyses directed caudally, parallel to spicules (Fig. 18ga), or absent . . . . . . . . 4 4

4 (3). Posterior part of buccal cavity collapsed, weakly cuticularized (Fig. 19bc); teeth present or absent ............ Comesomatinae

a. Teeth present at anterior end of buccal cavity; cuticle with or without distinct punctations (Figs. 77, 78). . . . . . Paracomesoma Hope \& Murphy, 1972

a'. Teeth absent at anterior end of buccal cavity; cuticle with distinct punctation ........b

b(a). Cuticle with lateral differentiation; gubernaculum with caudally directed apophyses (Figs. 19, 20) . . . . Comesomoides Gourbault, 1980

b'. Cuticle without lateral differentiation; gubernacular apophyses parallel to spicules (Fig.

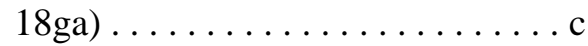

c(b). Outer labial and cephalic sensillae in single circle of 10 (Figs. 57cs, 58) . . . . . . . . .

Metacomesoma Wieser, 1954

$c^{\prime}$. Outer labial and cephalic sensillae in two separate circles (Figs. 17cs, 18) . . . . . . . . Comesoma Bastian, 1865

4'. Posterior part of buccal cavity expanded, heavily cuticularized (Fig. 42bc), teeth present or absent . . . . . . . . . Dorylaimopsinae

a. Teeth absent (Figs. 61bc, 62) . . . . . . . . Metasabatieria Timm, 1961

a'. Teeth present $\ldots \ldots \ldots \ldots \ldots \ldots \ldots$ b

b(a). Lateral differentiation present ...... c

b'. Lateral differentiation absent (Figs. 110, 111) ......... Vasostoma Wieser, 1954

c(b). Lateral differentiation of longitudinal rows of coarse punctuations (Figs. 261p, 27) . . . . . . . Dorylaimopsis Ditlevsen, 1918 
$c^{\prime}$. Lateral differentiation of transverse rows of coarse punctations............d

d(c). Posterior part of buccal cavity cylindrical (Figs. 42bc, 43) ... . . . Hopperia Vitiello, 1969

d'. Posterior part of buccal cavity conical (Figs. 85bc, 86) . . . . . P Paramesonchium Hopper, 1967

5 (2). Amphid unispiral (Fig. 28a); buccal cavity elongate, not funnel-shaped (Fig. 108bc) . . . . . . Ethmolaimidae

a. Extremely long somatic sensillae present; teeth equal (Figs. 108, 109) .... . Trichethmolaimus Platt, 1982

a'. Extremely long somatic sensillae absent; teeth unequal, dorsal tooth largest (Figs. 28, 29) . . . . . Ethmolaimus De Man, 1880

5'. Amphid multispiral; buccal cavity elongate, sometimes funnel-shaped . . . . . . . . 6

6 (5). Teeth mostly absent, mandibles present (Fig. 106m) or absent; if both teeth and mandibles absent, then cuticle with numerous longitudinal rows of small spines (Fig. 96sp) . . . . . Selachinematidae

a. Buccal cavity in two parts (Fig. 38); anterior part cup-shaped, posterior part cylindrical, both parts supported by cuticularized rods; mandibles absent . . b

a'. Buccal cavity not divided into two parts; mandibles present (Fig. 104m) or absent ..... g

$\mathrm{b}(\mathrm{a})$. Esophagus with distinct posterior bulb (Figs. 13, 14). ... . Choniolaimus Ditlevsen, 1918

b'. Esophagus without distinct posterior bulb . . c

c(b). Cephalic sensillae short, papilliform .....d

c'. Cephalic sensillae longer, setiform . . . . . . e e

d(c). Tail short, conical; tip blunt (Figs. 11, 12) . ... . . . . . . . Choanolaimus De Man, 1880

d'. Tail long, cylindrical then conical, sometimes flagellate (Figs. 40, 41) . . . . . . Halichoanolaimus De Man, 1886 e(c). Cuticle with 12-50 longitudinal rows of punctations (Figs. 51, 52ld) . . . . Latronema Wieser, 1954

e'. Cuticle without longitudinal rows of punctations ............. f

f(e). Tail short, conical (Figs. 38, 39) ....... Gammanema Cobb, 1920

f'. Tail long, flagellate (Figs. 15, 16) . . . . . . . Cobbionema Filipjev, 1922

g(a). Mandibles present .............

g'. Mandibles absent; cuticle with numerous longitudinal rows of small spines (Figs. 96sp, 97) ... ......... Richtersia Steiner, 1916

$\mathrm{h}(\mathrm{g})$. Mandibles equal in length (Fig. 104m) ... i

h'. Mandibles unequal in length, dorsally reduced (Fig. 24m) . . . . . . . . . j j

i(h). Mandibles single distally (Fig. 106m); cephalic sensillae papilliform; lateral differentiation absent; gubernaculum absent (Figs. 106, 107) . . . . . . .... Synonchium Cobb, 1920

i'. Mandibles doubled distally (Fig. 104m); cephalic sensillae setiform; lateral differentiation present; gubernaculum present (Figs. 104, 105) . . . . Synonchiella Cobb, 1933

j(h). Esophagus with distinct peribuccal bulb (9eb, 10) ......... Cheironchus Cobb, 1917

j'. Esophagus without distinct peribuccal bulb, tail variable . . . . . . . . . . k

$\mathrm{k}(\mathrm{j})$. Tail conical then filiform (Figs. 23-25) .... ......... Demonema Cobb, 1894

k'. Tail short, conical (Figs. 44, 45) . . . . . . . . .. Kosswigonema Gerlach, 1964

6'. Teeth, if present, appear as a single dorsal tooth and two subventral teeth (Fig. 71t) or a single dorsal tooth (Fig. 36t). If teeth absent then buccal cavity not divided into cup-shaped and cylindrical parts; mandibles absent .......... 7 
7 (6). Esophagus with posterior bulb; spicules typically bent about $2 / 3$ distance from proximal end (Fig. 70s); male precloacal supplements well developed, cup-shaped . . . . . . . Neotonchidae

a. Buccal cavity small, not heavily cuticularized (Fig. 67bc) ........... b

a'. Buccal cavity large, heavily cuticularized (Fig. 36bc) .............d

b(a). Labial sensillae setiform (Figs. 67, 68) . . . .......... Nannolaimus Cobb, 1920

b'. Labial sensillae papilliform (Fig. 301s) . . . . c c c(b). Spicules broad (Figs. 32, 33) Filitonchus Platt, 1982

c'. Spicules slender (Figs. 30, 31) Filitonchoides Jensen, 1986

d(a). Dorsal tooth massive (Fig. 36t); subventral teeth absent; spicules with lateral pieces (Figs. 36, 37) .... . Gomphionema Wieser \& Hopper, 1966

d'. Dorsal tooth not as massive; subventral teeth present or absent; spicules without lateral pieces ... e

e(d). Dorsal tooth extending anteriorly into vestibule; subventral teeth present, small (Figs. 34, 35). . . . . . . Gomphionchus Platt, 1982

$\mathrm{e}^{\prime}$. Dorsal tooth not extending anteriorly into vestibule (Fig. 71t); subventral teeth present or absent..........f

f(e). Dorsal and two small subventral teeth present (Figs. 71t, 72) . . . . . . . . Neotonchus Cobb, 1933

f'. Dorsal tooth present; subventral teeth reduced to ventral ridges (Figs. 69, 70) ..... Neotonchoides Platt, 1982

7'. Esophagus with or without a posterior bulb; spicules not characteristically bent about $2 / 3$ distance from proximal end; precloacal supplements cup-shaped, tubular or absent . . . . . . . . Cyatholaimidae.........8
8 (7). Ten longitudinal alae present; cuticular punctations absent (Figs. 112, 113) . . . . . . . Xenocyatholaimus Gerlach, 1953

8'. Longitudinal alae absent; cuticular punctations present ...............

9 (8). Precloacal supplements present in male ... ............... 10

9'. Precloacal supplements absent in male .... Cyatholaiminae (in part)

a. Esophagus with posterior bulb; teeth absent (Figs. 83, 84) ........ Paralongicyatholaimus Stekhoven, 1950

$a^{\prime}$. Esophagus without a posterior bulb; teeth present or absent ........... b

b(a). Dorsal tooth reduced or absent; lateral differentiation present (Figs. 59, 60 ) ........ Metacyatholaimus Stekhoven, 1942

b'. Dorsal tooth prominent (Fig. 22t); lateral differentiation absent ........... c

c(b). Lips leaf-like, bent backward (Figs. 87, 88) ........... Phyllolaimus Murphy, 1963

c'. Lips not leaf-like, not bent backward (Figs. 21, 22) . . . . . . . . Cyatholaimus Bastian, 1865

10 (9). Male precloacal supplements weakly cuticularized, simple cup-shaped (Fig. 6ps) or narrow tubes; lateral differentiation present......... Cyatholaiminae (in part)

a. Male precloacal supplements simple tubes (Figs. 93-95) .... Praeacanthonchus Micoletzky, 1924

a'. Male precloacal supplements small, cup-shaped (Fig. 6ps) . . . . . . . . . b

b(a). Buccal cavity with single dorsal tooth; subventral teeth as two pairs ........... c

b'. Buccal cavity with single dorsal tooth (Fig. 53t); subventral teeth absent or as a single pair .....d 
c(b). Hypodermal pore complexes present in eight longitudinal rows (Figs. 114-115) . . . . Xyzzors Inglis, 1963

c'. Pores arranged in 12 longitudinal rows, reduced in area between nerve ring and preanal region (Figs. 55-56) . . . . Marilynnia Hopper, 1972

d(b). Lateral differentiation present (Figs. 53, 54) . . . . . Longicyatholaimus Micoletzky, 1924

d'. Lateral differentiation absent (Figs.5-6) . . . . . .............. Biarmifer Wieser, 1954

10'. Male precloacal supplements strongly cuticularized, complex cup-shaped (Fig. 64ps), tubular (Fig. 2ps) or setose; lateral differentiation present or absent .......... 11

11 (10). Male precloacal supplements protrusible tubes (Fig. 2ps) or setose (Fig. 80ps) .... . Paracanthonchinae

a. Male precloacal supplements protrusible tubes ..............b

a'. Male precloacal supplements setose ...... c

b(a). Gubernaculum dentate (Fig. 75g) (Figs. 73-76) . . . . Paracanthonchus Micoletzky, 1924

b'. Gubernaculum not dentate (Figs. 1, 2) . . . . . . .......... Acanthonchus Cobb, 1920

c(a). Amphids of both sexes dissimilar in size (Figs. 81, 82) .... Paracyatholaimoides Gerlach, 1953

c'. Amphids of both sexes similar in size (Figs. 79, 80). . . . Paracyatholaimus Micoletzky, 1922

11'. Male precloacal supplements complex, consisting of a number of elements (64ps) . . . . . . . .... Pomponematinae

a. Buccal cavity large with teeth (Fig. 46t). . . b

a'. Buccal cavity small without teeth (Figs. 63, 64) . . . . . . . . . . . Minolaimus Vitiello, 1970

$b(a)$. Cuticle with eight longitudinal rows of punctations (Figs. 46-48) . . . . . . . . .

Kraspedonema Gerlach, 1954 b'. Cuticle without eight longitudinal rows of punctations ............. c

c(b). Buccal cavity with large dorsal tooth and two subventral teeth; denticles may also be present; esophageal bulb absent (Figs. 91, 92) . . . . . . Pomponema Cobb, 1917

c'. Buccal cavity with dorsal and subventral teeth reduced; denticles absent; esophageal bulb present (Figs. 65-66) . . . . Nannolaimoides Ott, 1972

\section{Acknowledgments}

We express our sincere appreciation to Drs. K. B. Nguyen, R. P. Esser, and especially G. C. Smart, Jr., for their review of this manuscript and suggestions for its improvement.

\section{Selected References}

Andrassy, I. 1976. Evolution as the basis for the systematization of nematodes. Pitman Publishing, London. Akademiai Kaido, Budapest, 288 p.

Gerlach, S.A., and Riemann, F. 1973. The Bremerhaven checklist of aquatic nematodes. Veröff. Inst. Meeresforsch. Bremerhaven, Supplement 4, p. 1-734.

Hope, W.D., and Murphy, D.G. 1972. A taxonomic heirarchy and checklist of the genera and higher taxa of marine nematodes. Smithson. Contrib. Zool. 137, p. 1-101.

Inglis, W.G. 1983. An outline classification of the phylum Nematoda. Aust. J. Zool. 31:243-255.

Jensen, P. 1979. Revision of the Comesomatidae (Nematoda). Zool. Scripta 8:81- 105.

Lorenzen, S. 1981. Entwurf eines phylogenetischen Systems der freilebenden Nematoden. Veröff. Inst. Meeresforsch. Bremerhaven, Supplement 7, p. 1-472.

Platt, H.M. 1982. Revision of the Ethmolaimidae (Nematoda: Chromadorida). Bull. Brit. Mus. (Nat. Hist.) Zool. 43:185-252. 
Platt, H.M., and Warwick, R.M. 1983. Free-living marine nematodes. Part I. British Enoplids. Cambridge Univ. Press, Cambridge, 307 p.

Platt, H.M., and Warwick, R.M. 1988. Free-living marine nematodes. Part III. British Chromadorids. E. J. Brill/Dr. W. Backhuys, New York, 502 p.

Tarjan, A.C. 1980. An illustrated key to the marine nematodes. Inst. Food Agric. Sci., Univ. Florida, Gainesville, 135 p.

\section{References for Illustrations}

Boucher, G. 1976. Nématodes dessables fins infralittoraux de la Pierre Noire (Manche accidentale) II. Chromadorida. Bul. Mus. Nat. d'Hist. Nat. Paris, 3e ser, no. 352 Zool. 245:25-61.

Furstenberg, J.P., and Heyns, J. 1987. Cobbionema capense $\mathrm{n}$. sp. from Swartkops estuary, Port Elizabeth (Nematoda: Selachinematidae). Can. J. Zool. 65:80-82.

Gerlach, S.A. 1953a. Die Nematodenbesiedlung des Sandstrandes und des Kustengrundwassers an der italienischen Kuste. I. Systematischer Teil. Arch. Zool. Italiano 37:517-640.

Gerlach, S.A. 1953b. Recherches sur la fauna des eaux interstitielles de Madagascar. III. Mem. Instit. Scient. Madagascar Ser. A. 8:73-86.

Gerlach, S.A. 1954. Nouveaux nématodes libres des eaux souterraines littorales Francaises. Vie et Milieu 4:94-110.

Gerlach, S.A. 1956. Diagnosen neuer Nematoden aus der Kieler Bucht. Kieler Meeresforsch. 12:85-109.

Gerlach, S.A. 1958. Freilebende Nematoden von den Korallenriffen des Roten Meeres. Kieler Meeresforsch. 14:241-246.

Gerlach, S.A. 1964a. Revision der Choniolaiminae und Selachinematinae (freilebende Meeres Nematoden). Mitt. Hamburg. Zool. Mus. Inst. Kosswig-Festschrift, pp. 23-50.
Gerlach, S.A. 1964c. Neue Cyatholaimidae (Nematoda, Chromadorida) von den Malediven. Veröff. Inst. Meeresforsch. Bremerhaven 9:70-78.

Gourbault, N. 1980. Nématodes abyssaux (Campagne Walda du N/O << J. Charcot $>$ ). II. Espèces et genre nouveaux de Comesomatidae. Bull. Mus. natn. Hist. nat., Paris 4 ser. 2:737-749.

Hopper, B.E. 1967. Free-living marine nematodes from Biscayne Bay, Florida. I. Comesomatidae: the male of Laimella longicauda Cobb, 1920, and a description of Actarjania new genus. Mar. Biol., Int. J. Life Oceans Coast. Waters 1:140-144.

Hopper, B.E. 1972. Free-living marine nematodes from Biscayne Bay, Florida. IV. Cyatholaimidae: On the occurrence of Marilynia $\mathrm{n}$. gen. and Longicyatholaimus Micoletzky, 1924 in Biscayne Bay, with a description of L. longicaudatus (de Man, 1876) from the type locality. Zool. Anz., Leipzig 189:64-88.

Inglis, W.G. 1963. New marine nematodes from off the coast of South Africa. Bull. Brit. Mus. nat. Hist. (Zool.) 10:529-552.

Inglis, W.G. 1970. Cyatholaimidae (Nematoda) from the coast of Western Australia. Rec. S. Aust. Mus. 16:1-13.

Jensen, P. 1976. Free-living marine nematodes from a sublittoral station in the North Sea off the Belgian coast. Biol. Jb. Dodonaea 44:231-255.

Jensen, P. 1979. Nematodes from the brackish waters of the southern archipelago of Finland. Benthic species, Ann. Zool. Fennici 16:151-168.

Jensen, P. 1986. The nematode fauna in the sulphide-rich brine seep and adjacent bottoms of the East Flower Garden, NW Gulf of Mexico. I. Chromadorida. Zool. Scripta 14:247-263.

Jensen, P., and Gerlach, S.A. 1977. Three new Nematoda - Comesomatidae from Bermuda. Ophelia 16:59-76.

Murphy, D.G. 1963. A new genus and two new species of nematodes from Newport, Oregon. Proc. Helm. Soc. Wash. 30:73-78. 
Murphy, D.G. 1964. Free-living marine nematodes, I. Southerniella youngi, Dagda phinneyi, and Gammanema smithi, new species. Proc. Helm. Soc. Wash. 31:190-198.

Ott, J.A. 1972. Twelve new species of nematodes from an intertidal sandflat in North Carolina. Int. Revue ges. Hydrobiol. 57:463-496.

Pastor de Ward, C.T. 1979. Free-living marine nematodes (subclass Adenophorea) of the Ria Deseado (Santa Cruz, Argentina). Ann. Soc. Roy. Zool. Belg. 108:29-45.

Platt, H.M. 1982. Revision of the Ethmolaimidae (Nematoda: Chromadorida). Bull. Brit. Mus. (Nat. Hist.) Zool. Ser. 43:185-252.

Platt, H.M. 1985. The freeliving marine nematode genus Sabatieria (Nematoda: Comesomatidae). Taxonomic revision and pictorial keys. Zool. J. Linnean Soc. 83:27-78.

Riemann, F. 1966. Die interstitielle Fauna im Elbe-Aestuar Verbreitung und Systematik. Arch. Hydrobiol. Suppl. XXXI:1-279.

Schuurmans Stekhoven, J.H. 1950. The free-living marine nemas of the Mediterranean. I. The bay of Villefranche. Mem. Inst. Roy. Sci. Nat. Belg. 37:1-220.

Sergeeva, N.G. 1973. New species of free-living marine nematodes from the order Chromadorida in the Black Sea. Zool. Zh. 52:1238-1241.

Soetaert, K., and Vincx, M. 1987. Six new Richtersia species (Nematoda, Selachinematidae) from the Mediterranean Sea. Zool. Scripta 16:125-142.

Timm, R.W. 1961. The marine nematodes of the Bay of Bengal. Proc. Pakistan Acad. Sci. 1:1-88.

Vitiello, P. 1969. Hopperia, nouveau genre de nématode libre marin (Comesomatidae). Tethys 1:485-491.

Vitiello, P. 1970. Nématodes libres marins des vases profondes du Golfe du Lion. II. Chromadorida. Tethys 2:449-500.
Warwick, R.M. 1971. The Cyatholaimidae (Nematoda, Chromadoroidea) off the coast of Northumberland. Cah. Biol. Marine 12:95-110.

Wieser, W. 1954. Free-living marine nematodes. II. Chromadoroidea. Acta Univ. Lund Sect. II Med. Math. Sci. Rerum Nat. 50:1-148.

Wieser, W. 1955. A collection of marine nematodes from Japan. Publ. Seto. Mar. Biol. 4:159-181.

Wieser, W. 1959. Free-living nematodes and other small invertebrates of Puget Sound beaches. Univ. Washington Publ. Biol. 19:1-179.

Wieser, W., and Hopper, B.E. 1966. The Neotonchiinae, new subfamily (Cyatholaimidae: Nematoda), with an analysis of its genera, NeotonchusCobb, 1933 and Gomphionema new genus. Can. J. Zool. 44:519-532.

Wieser, W., and Hopper, B.E. 1967. Marine nematodes of the East coast of North America. I. Florida. Bull. Mus. Comp. Zool. 135:239-344. 
Illustrated Key to the Genera of Free-Living Marine Nematodes in the Superfamily....

Table 1. Alphabetical List of Genera and Illustration Sources

\begin{tabular}{|c|c|c|}
\hline Figure No. & Genus & Illustration Source \\
\hline $1-2$ & Aconthonchus & Wieser 1955 , p. 66 , Figs. a, b \\
\hline $3-4$ & Actarjania & Hopper 1967, p. 141, Figs. 5,7 \\
\hline $5-6$ & Biarmifer & Wieser 1954 , p. 11 , Figs. c, e \\
\hline 7-8 & Cervonema & Gourbault 1980, p. 739, Figs. 1c, d \\
\hline $9-10$ & Cheironchus & Gerlach 1964a, p. 46, Figs. a, b \\
\hline $11-12$ & Choanolaimus & Riemann 1966, p. 133, Figs. 34a, c \\
\hline $13-14$ & Choanolaimus & Riemann 1966, p. 110, Figs. b, d \\
\hline $15-16$ & Cobbionema & Furstenberg \& Heyns 1987, p. 81, Figs. 1,4 \\
\hline 17-18 & Comesoma & Wieser 1954a, p. 137, Figs. 177b, d \\
\hline $19-20$ & Comesomoides & Gourbault 1980, p. 747 , Figs. 4a, c \\
\hline $21-22$ & Cyatholaimus & Wieser 1959, PI. XV, Fig. 37a; PI. XVI, Fig. 37b \\
\hline $23-25$ & Demonema & Gerlach 1958, Taf. 40, Figs. 1b, c, d \\
\hline $26-27$ & Dorylaimopsis & Schuurmans Stekhoven 1950, p. 147, Figs. 88A, I \\
\hline $28-29$ & Ethmolaimus & Jensen 1979 , p. 165, Figs. 57, 58 \\
\hline $30-31$ & Filitonchoides & Jensen 1986, p. 253, Figs. 6B, E \\
\hline $32-33$ & Filitonchus & Platt 1982, p. 248, Figs. 36b, d \\
\hline 34 & Gomphionchus & Wieser \& Hopper 1966, p. 527, Figs. 20, 21 \\
\hline 35 & Gomphionchus & Platt 1982, p. 217, Figs. 14a, b \\
\hline $36-37$ & Gomphionchus & Wieser \& Hopper 1966, p. 530, Figs. 31, 32 \\
\hline 38-39 & Gammanema & Murphy 1964, p. 195, Fig. 3a; p. 196, Fig. 4 \\
\hline $40-41$ & Halichoanolaimus & Wieser \& Hopper 1967, p. 324, Figs. 34A, 35B \\
\hline $42-43$ & Hopperia & Vitiello 1969, p. 486, Fig. 1b; p. 488, Fig. 2a \\
\hline $44-45$ & Kosswigonema & Gerlach 1964a, p. 45, Figs. 15a, e \\
\hline $46-48$ & Kraspedonema & Gerlach 1954, p. 100, Figs. 2b, c, d \\
\hline $49-50$ & Laimella & Hopper 1967, p. 144, Figs. 2, 3 \\
\hline $51-52$ & Latronema & Wieser 1954, p. 31, Figs. 109a, d \\
\hline $53-54$ & Longicyatholaimus & Vitiello 1970, p. 476 , Figs. 18A, D \\
\hline $55-56$ & Marilynia & Hopper 1972, p. 75 , Figs. 14,15 \\
\hline $57-58$ & Metacomesoma & Wieser 1954, p. 137, Figs. 176a, c \\
\hline $59-60$ & Metacyatholaimus & Gerlach 1964b, p. 73, Figs. 2b, d \\
\hline 61-62 & Metasabatieria & Timm 1961, p. 51, Figs. 35a, b \\
\hline $63-64$ & Minolaimus & Vitiello 1970 , p. 486 , Figs. $25 \mathrm{~b}, \mathrm{f}$ \\
\hline $65-66$ & Nannolaimoides & Ott 1972, p. 464, Figs. 4,7 \\
\hline $67-68$ & Nannolaimus & Platt 1982, p. 242, Figs. 32b, d \\
\hline $69-70$ & Neotonchoides & Platt 1982, p. 238, Fig. 29c \\
\hline $71-72$ & Neotonchus & Platt 1982, p. 220, Figs. 16a, b \\
\hline 73 & Paracanthonchus & Wieser \& Hopper 1967, p. 323, Fig. 31a \\
\hline 74 & Paracanthonchus & Inglis 1970 , p. 7 , Fig. 13 \\
\hline $75-76$ & Paracanthonchus & Wieser \& Hopper 1967, p. 323, Figs. 31b, c \\
\hline 77-78 & Paracomesoma & Jensen \& Gerlach, 1977, p. 61, Figs. 1a, c \\
\hline $79-80$ & Paracyatholaimus & Wieser \& Hopper 1967, p. 323, Figs. 32a, b \\
\hline $81-82$ & Paracyatholaimoides & Boucher 1976, p. 48, Figs. 7c, f \\
\hline $83-84$ & Paralongicyatholaimus & Warwick 1971, p. 101, Figs. 3a, d \\
\hline $85-86$ & Paramesonchium & Jensen 1976, p. 245 , Figs. 30,36 \\
\hline $87-88$ & Phyllolaimus & Murphy 1963, p. 76 , Figs. 2A, C \\
\hline $89-90$ & Pierrichia & Vitiello 1970 , p. 455, Figs. 3A, D \\
\hline
\end{tabular}


Illustrated Key to the Genera of Free-Living Marine Nematodes in the Superfamily....

Table 1. Alphabetical List of Genera and Illustration Sources

\begin{tabular}{||l|l|l||}
\hline \hline \multicolumn{1}{|c|}{ Figure No. } & \multicolumn{1}{|c||}{ Genus } & \multicolumn{1}{c||}{ Illustration Source } \\
\hline $91-92$ & Pomponema & Wieser \& Hopper 1967, p. 321, Figs. 28a, c \\
\hline $93-95$ & Praecanthonchus & Pastor de Ward 1979, p. 35, Figs. 5b,g, e \\
\hline $96-97$ & Richtersia & Soetaert \& Vincx 1986, p. 129, Fig. 3b \\
\hline $98-99$ & Sabatieria & Wieser \& Hopper 1967, p. 335, Figs. 59a, c \\
\hline $100-101$ & Scholpaniella & Sergeeva 1972, p. 1293, Figs. 5, 6 \\
\hline $102-103$ & Setosabatieria & Platt 1985, p. 43, Fig. 9 \\
\hline $104-105$ & Synonchiella & Riemann 1966, p. 135, Figs. 36a, d \\
\hline $106-107$ & Synonchium & Gerlach 1953, p. 556, Figs. 18a, c \\
\hline $108-109$ & Trichethmolaimus & Gerlach 1956, Taf. 31, Figs. a, b \\
\hline $110-111$ & Vasostoma & Wieser 1954a, p. 137, Figs. 175a, b \\
\hline $112-113$ & Xenocyatholaimus & Gerlach 1953, p. 74, Figs. 1a, c \\
\hline $114-115$ & Zyzzors & Inglis 1963, p. 545, Fig. 25 \\
\hline & Wieser \& Hopper 1967, p. 322, Fig. 29 \\
\hline \hline
\end{tabular}

